\title{
MADERA CARBONIZADA EN CONTEXTOS FUNERARIOS DE LA JEFATURA DE RÍO GRANDE, PANAMÁ: ANTRACOLOGÍA EN EL SITIO DE EL CAÑO
}

\author{
CARBONISED WOOD IN FUNERARY CONTEXTS OF THE RÍO GRANDE \\ CHIEFDOM, PANAMA: CHARCOAL ANALYSIS AT THE SITE OF EL CAÑO
}

\author{
María Martín Seijo ${ }^{1}$, Raquel Piqué i Huerta², Julia Mayo Torné3,4, \\ Carlos Mayo Torné1, 3, 5 y Emilio Abad Vidal ${ }^{6}$
}

\begin{abstract}
El análisis antracológico realizado en el sito de El Caño (provincia de Coclé, Panamá) nos permite hacer una primera aproximación a las posibilidades que este tipo de análisis arqueobotánico proporciona para el estudio de las sociedades de jefatura, y específicamente de sus contextos funerarios. La identificación de los recursos leñosos utilizados, y el establecimiento de hipótesis acerca de su posible función en relación con el ritual mortuorio, es fundamental para poder profundizar en la comprensión del ritual y de la gestión de los recursos realizada por las jefaturas en el istmo de Panamá.
\end{abstract}

Palabras claves: antracología, arqueobotánica, jefaturas, contextos funerarios, recursos leñosos.

The charcoal analysis undertaken at the El Caño site (Coclé Province, Panama) provided an opportunity to explore the possibilities this kind of archaeobotanical analysis supplies for the study of chiefdoms, particularly in their funerary context. The identification of the utilization of wood resources, and the establishment of a hypothesis about their possible funerary ritual function is fundamental in order to deepen our understanding of the ritual and the management of resources by chiefdoms in the Isthmus of Panama.

Key words: Charcoal analysis, Archaeobotany, chiefdoms, funerary contexts, wood resources.

Este artículo presenta los resultados de la identificación taxonómica y estudio dendrológico de los carbones recuperados en el sitio de El Caño, anteriormente conocido por sus singulares esculturas y sus estructuras arqueológicas en piedra, hasta que en los últimos años han sido localizadas varias tumbas de individuos con ricos ajuares datadas entre el 750 y el 1.020 d.C. (Mayo y Carles 2015; Mayo y Mayo 2013).

El estudio de carbones y maderas recuperados en contextos arqueológicos ha tenido un gran desarrollo en las últimas décadas en América Central, aunque con una aplicación desigual, concentrándose la mayor parte en el área mesoamericana: Sur de México, Belize y Guatemala (Cavallaro 2013; Hood 2012; Lentz et al. 1996; Lentz y Hockaday 2009; McKillop 2014, 2013; Morehart y Morell-Hart 2013; Morehart et al. 2005; Prufer y Dunham 2009; Robinson y McKillop 2005; Wyatt 2008). En las Antillas su aplicación también es dispar, agrupándose los análisis antracológicos en Haití, Puerto Rico y Bahamas (Berman y Pearsall 2000; Newsom y Wing 2004;

1 GEPN-Grupo de Estudos para a Prehistoria do NW Ibérico (GI-1534). Departamento de Historia I. Universidade de Santiago de Compostela. Autora correspondiente: María Martín Seijo, Facultade de Xeografía e Historia. Praza da Universidade s/n. 15782 Santiago de Compostela, España.

maria.martin.seijo@gmail.com

2 Departament de Prehistòria, Universitat Autònoma de Barcelona. 08193 Bellaterra, España. raquel.pique@uab.cat

3 Centro de Investigaciones Arqueológicas del Istmo, Fundación El Caño, APO 819-4446, El Dorado, Panamá, República de Panamá.

juliamayo@fundacionelcano.org; carlosmayo@fundacionelcano.org

4 Center for Tropical Archaeology and Paleoecology. Smithsonian Tropical Research Institute. Republic of Panama. mayoj@si.edu

5 Dirección Nacional de Patrimonio Histórico, Panamá.

6 CESGA-Fundación Centro Tecnolóxico de Supercomputación de Galicia, 15705, Santiago de Compostela, España. eav@cesga.es

Recibido: febrero 2015. Aceptado: noviembre 2015.

http://dx.doi.org/10.4067/S0717-73562016005000013. Publicado en línea: 6-abril-2016. 
Pagán 2002), aunque para esta área disponemos también de identificaciones realizadas sobre objetos de madera (Carreras 2009; Ostapkowicz et al. 2012; Ostapkowicz, Bronk Ramsey, Brock et al. 2011; Ostapkowicz, Wiedenhoeft, Bronk Ramsey et al. 2011; Saunders y Gray 1996).

En lo que respecta al norte de Colombia y de Venezuela, solo en la primera se han realizado análisis de maderas carbonizadas (Archila 2008; Archila y Cavelier 2006). Mientras que en Panamá la antracología ha sido una disciplina aplicada de forma muy esporádica (Archila 2008:82), ya que la investigación de restos vegetales ha privilegiado el estudio de otro tipo de evidencias relacionadas con el estudio del procesado y consumo de alimentos o con indicadores de la evolución de la cubierta vegetal.

Los datos antracológicos de El Caño permiten una aproximación al uso de la madera entre las sociedades de jefatura del istmo de Panamá. Las referencias a la explotación de los recursos forestales y a los usos de la madera en diferentes ámbitos de la vida de estas comunidades se habían hecho hasta el momento de forma indirecta utilizando referencias etnográficas (Helms 1979) o documentación etnohistórica (Cooke 1998). Este estudio arqueobotánico supone dar visibilidad al uso de los recursos forestales por parte de estas comunidades como combustible o como madera utilizada para la construcción de edificaciones, la elaboración de objetos domésticos, artesanales o rituales, la confección de armas, canoas, etc. (Cooke y Sánchez 2004). La invisibilidad de la madera y de otros elementos vegetales en las investigaciones arqueológicas está relacionada fundamentalmente con aspectos metodológicos, ya que la inexistencia de estrategias de recolección sistemática de muestras arqueobotánicas durante las intervenciones arqueológicas impide disponer de material representativo para estudio.

$\mathrm{Al}$ abordar la investigación de la madera en esta área debemos tener presente que los árboles eran percibidos por las comunidades amerindias con cosmologías animistas como seres vivos equiparables a los seres humanos, con un ciclo vital similar-desde el nacimiento, pasando por la madurez y la muerte-, que equiparaban además su sabia a la sangre y su estructura al cuerpo humano. Para estas comunidades, las diferencias entre seres humanos, plantas y animales eran diferencias de grado y no de naturaleza (Viveiros de Castro 1996; Descola 1998). Probablemente los árboles que eran parte de su entorno y también de esta cosmología, sobre todo aquellos que desarrollaban un papel simbólico y/o práctico destacado (Heyden 1993). En este sentido, los árboles que eran considerados sagrados se caracterizaban por poseer un importante valor económico y múltiples utilidades para las comunidades, llegando a mezclarse en ocasiones a las creencias y los usos de las mismas. Es importante señalar además que las propiedades atribuidas a los árboles se extienden también a la madera extraída de estos e incluso a los objetos elaborados a partir de las mismas (Saunders y Gray 1996; Orliac 1990-1).

La presencia de madera en los contextos funerarios era probablemente extensa, tanto en lo que respecta a la construcción y adecuación de la tumba como en los objetos depositados como ofrendas y combustibles utilizados en las diferentes fases del ritual. Esto hace que para comprender en su complejidad estos ritos sea imprescindible abordar el estudio de la madera preservada en contextos funerarios. A un nivel más general la identificación de las especies leñosas y la interpretación de su uso nos proporcionarán nuevos datos respecto del manejo de los recursos forestales por las sociedades de jefatura, incluyendo la posibilidad de que existan interacciones regionales y comercio de madera $u$ objetos elaborados en este material. Visibilizar los ámbitos del ritual funerario en los que estaba presente la madera puede proporcionar también información acerca del acceso a los recursos, el simbolismo de determinados árboles y de los postes de madera confeccionados a partir de estos, etc.

\section{El Caño}

El Caño se encuentra situado a $50 \mathrm{msm}$ en la vertiente pacífica de Panamá, en una llanura aluvial que se inunda periódicamente por el desbordamiento del río Grande que desemboca en la bahía de Parita (Figura 1). En esta zona con clima tropical de sabana (Awi) la temperatura promedio oscila entre 26,35 y $29,25{ }^{\circ} \mathrm{C}$, con una precipitación máxima anual de $1.216,2 \mathrm{~mm}$ y cinco meses con precipitación menor de $30 \mathrm{~mm}$. La vegetación que crece actualmente en el entorno del yacimiento está fuertemente alterada y antropizada, permaneciendo en el área arqueológica solo unos pocos árboles (Figura 2). En las inmediaciones se encuentran una zona de potreros, grandes fincas destinadas al cultivo de caña, arroz y cebolla, junto con pequeñas huertas. 


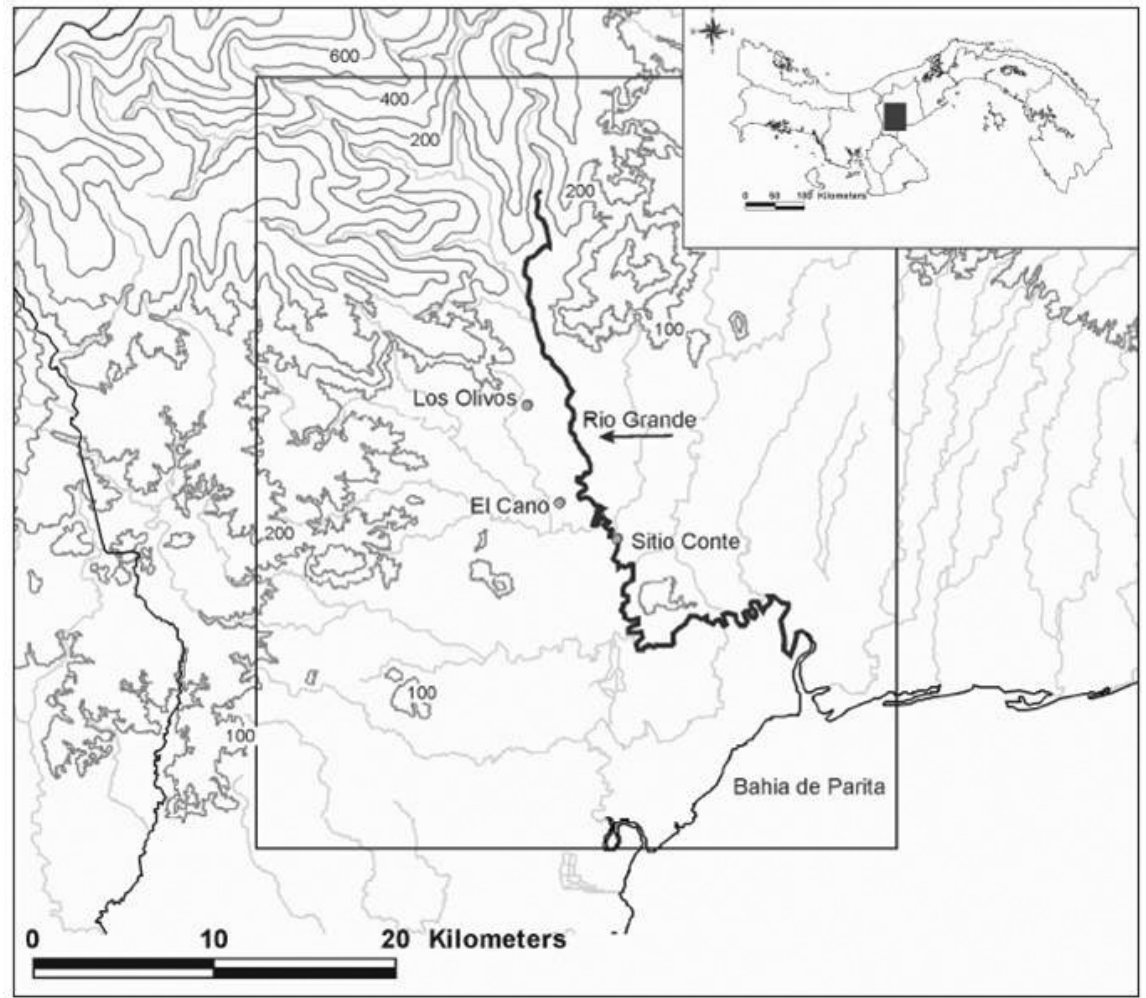

Figura 1. Localización del sitio arqueológico en el istmo de Panamá.

Location of the archaeological site in the Isthmus of Panama.

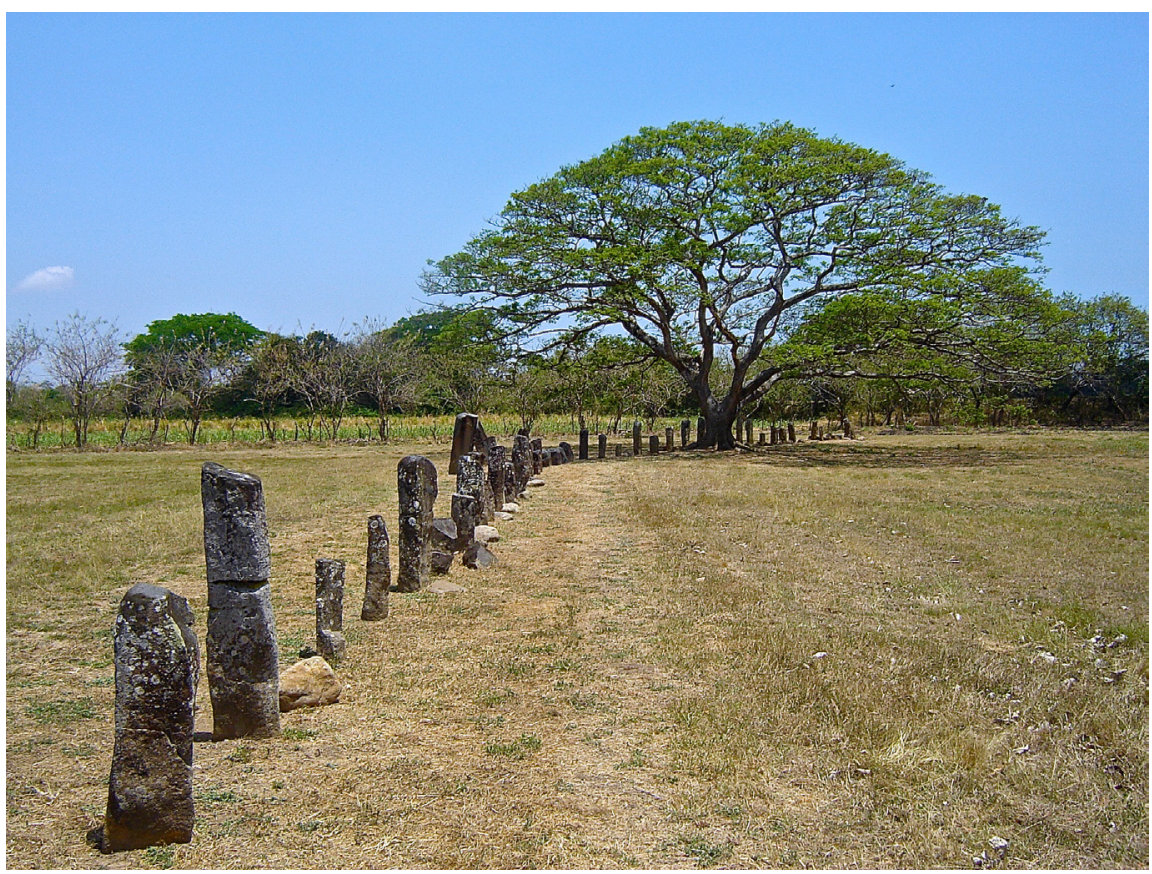

Figura 2. Alineamientos de columnas basálticas y paisaje antropizado en el entorno de El Caño. Alignments of basalt columns and the anthropized landscape in the environs of El Caño. 
La vegetación potencial de esta área es el bosque seco tropical que ha sido hoy prácticamente despojado de su cobertura forestal original, excepto en los manglares estuarios y entradas costeras. En estos bosques secos predominan los árboles caducifolios, con copas y áreas basales más bajas que los bosques tropicales lluviosos, y presencia significativa de especies espinosas (Murphy y Lugo 1986; Pennington et al. 2009). La vegetación leñosa está dominada por especies de la familia de las Fabaceae y se encuentran también representadas especies de las siguientes familias: Anacardiaceae, Burseraceae, Myrtaceae, Sapindaceae y Capparaceae (modificado a partir de Pennington et al. 2006; Condit et al. 2011). Los manglares en los estuarios y ensenadas costeras incluyen especies de las familias Acanthaceae, Combretaceae, Pellicierae y Rizhophoraceae.

En las inmediaciones del valle fluvial del río Grande se encuentran algunos de los yacimientos arqueológicos precolombinos más importantes del istmo, entre ellos varios pequeños asentamientos como Rancho Sancho, Natá y Cerrezuela (Cooke 1972; Mayo et al. 2007), y varios complejos funerarios como Sitio Conte (Lothrop 1937), Los Olivos y El Caño (Mayo y Carles 2015; Mayo y Mayo 2013).

Las intervenciones arqueológicas efectuadas en El Caño han sido numerosas, remontándose las primeras a 1926 (Verryl 1927) y sucediéndose hasta finales de la década de 1980 (Fitzgerald 1992). Estos primeros trabajos arqueológicos permitieron caracterizar este sitio inicialmente como un centro ceremonial relacionado con la necrópolis de Sitio Conte, considerada hasta el momento el único lugar de entierro de élite de la región (Cooke 2004a, 2004b). Sin embargo las últimas intervenciones arqueológicas realizadas entre 2008 y 2014 han permitido identificarlo como un cementerio independiente donde fueron enterrados algunos miembros de la élite de la jefatura de Río Grande entre el 750-1.020 d.C. (Mayo y Carles 2015; Mayo y Mayo 2013) (Figura 3). La cercanía de dos cementerios de élite coetáneos y el hallazgo en El Caño de entierros de infantes ataviados con ricos ajuares están cambiando la perspectiva que hasta este momento se tenía respecto de la extensión y complejidad de esta jefatura (Mayo y Carles 2015; Mayo y Mayo 2013).

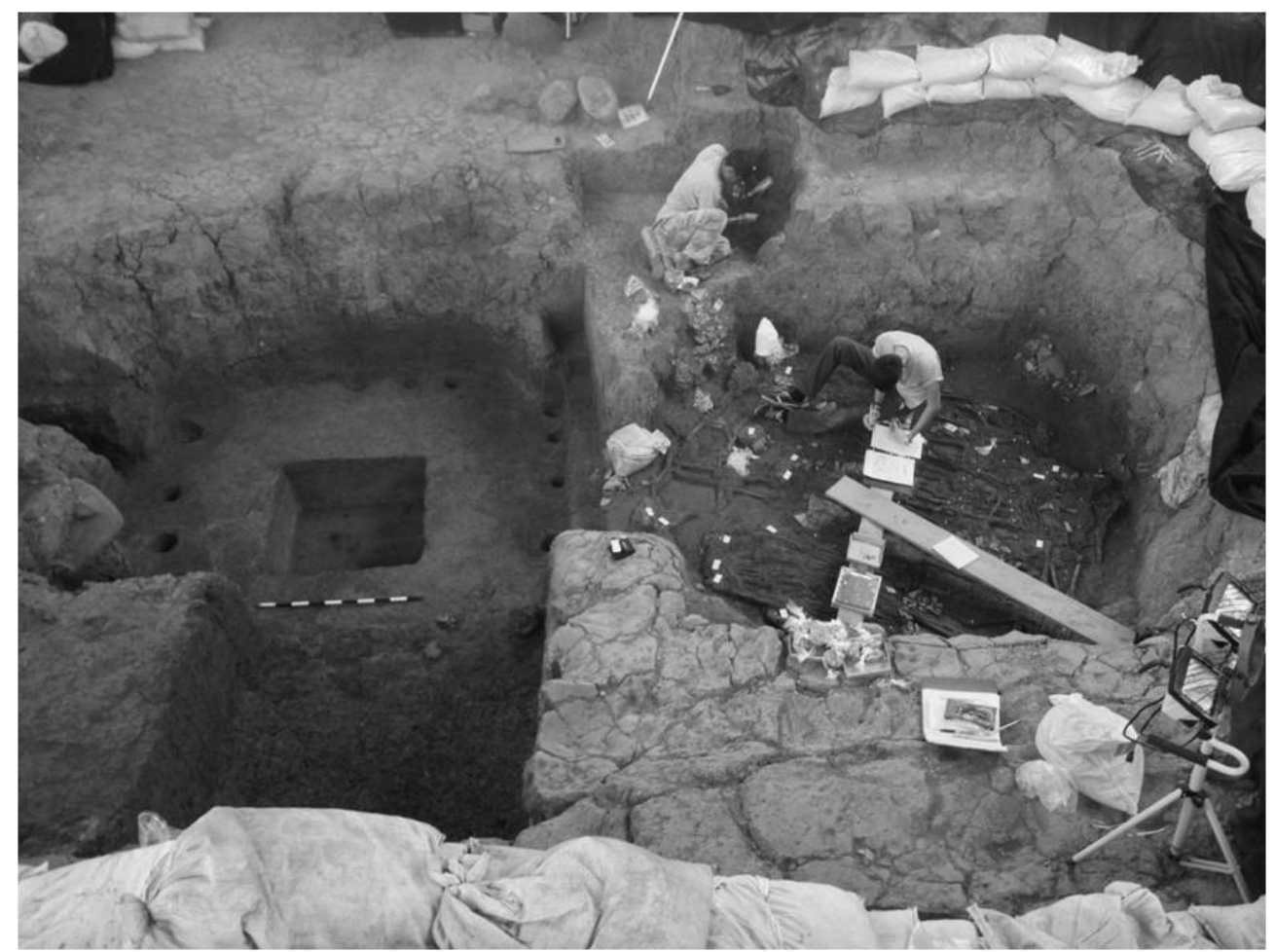

Figura 3. Imagen del proceso de excavación de las tumbas.

Image of the tombs' excavation process. 
Los datos disponibles hasta el momento indican que la "Jefatura del Río Grande" fue la más compleja de la región cultural del Gran Coclé, probablemente por el control que las comunidades de esta área ejercieron sobre los recursos naturales de su entorno, incluyendo el oro o el cobre, y el control que practicaron sobre el sistema de intercambio, ya que el valle del río Grande se encuentra en el centro de una región con una rica y variada oferta de recursos naturales y con una posición estratégica para el control del territorio, al estar bien comunicada por vía fluvial y marítima. Respecto del estatus social de los individuos enterrados en El Caño, las últimas intervenciones han permitido documentar cómo se produce un cambio social hacia el 900 d.C. cuando los estatus sociales se hacen hereditarios, frente a la fase inicial en la que el poder de los líderes estaba todavía ligado al prestigio. En definitiva, el estudio de los artefactos y muestras encontradas dentro de las tumbas de este yacimiento permitirán definir de un modo más preciso las características de las jefaturas del istmo entre el 750 y el 1.020 d.C.

\section{El Ritual Funerario}

El ritual funerario de las Jefaturas de la bahía de Parita ha sido uno de los temas fundamentales de estudio y debate sobre la jerarquía social en las Américas, y es conocido a partir de las intervenciones arqueológicas desarrolladas en Sitio Conte (Linares 1977; Lothrop 1934, 1937) y El Caño (Mayo y Carles 2015; Mayo y Mayo 2013), así como por los datos etnohistóricos recuperados a partir de las descripciones recogidas en los escritos de Pascual de Andagoya, Gonzalo Fernández de Oviedo y Gaspar de Espinosa. Debemos tener presente que estas descripciones son posteriores al período de funcionamiento de ambas necrópolis, por lo que los datos contenidos en las mismas deben ser tomados con precaución (Linares 1977).

Las jefaturas se caracterizan principalmente por presentar una marcada jerarquía social y por la acumulación de riqueza en manos de unos pocos individuos. Esto es especialmente evidente en los contextos funerarios de El Caño y Sitio Conte en los que cientos de artefactos de prestigio, muchos de ellos con iconografía cosmológica, formaban parte del ajuar funerario de los individuos de alto rango (Linares 1976; Lothrop 1937; Mayo y Mayo 2013; Menzies y Haller 2012). Probablemente las tumbas de ambos yacimientos contenían, entre otros objetos, artefactos de madera como duhos, astiles de flechas, lanzas y atlatls. De algunos podemos deducir su presencia en los ajuares a partir de la conservación de los propulsores y de algunos artefactos con los que reforzaban las puntas de las lanzas como puntas de piedra, arpones de hueso, espinas caudales de rayas y dientes de tiburón perforados. También en madera eran elaborados algunos objetos de adorno personal como las orejeras (Mayo y Mayo 2013:13). Este tipo de elementos perecederos solo se conservan en caso de haber entrado en contacto con el fuego, este fue el caso de objetos de madera y cestería carbonizados recuperados en las tumbas de Sitio Conte (Lothrop 1937:49).

También está presente la madera en la construcción de diferentes elementos de las tumbas. Oviedo describe la existencia de construcciones de madera, con paredes de caña y cubierta vegetal denominadas buhíos o bohíos que las cubrirían (Fernández de Oviedo III, XXIX, XXXI:453-456). Las características de estas construcciones aparecen también recogidas en el Sumario de la Natural Historia de las Indias (1526) (Fernández de Oviedo III, XXIX, XXVII:131-132), y son comunes a las de otras zonas del istmo, como podemos observar en la descripción de A. Reclus ya en el siglo XIX para la zona del Darién (Reclus 1881:176-177). En las tumbas 1 y 2 de El Caño se localizaron los restos de cubiertas de madera carbonizadas y restos de los pilares que las sostenían (Mayo y Mayo 2013).

Respecto del ritual funerario, Gaspar de Espinosa hace referencia al tratamiento del cuerpo del cacique Antatará en Parita, describiendo cómo estas comunidades no entierran a sus jefes sino que los mantienen en una casa especial, preservando sus cuerpos colocándolos sobre unos bancos de piedra y secándolos con fuegos mantenidos durante varios días, o en otros casos envolviendo el cuerpo en cinco o seis mantas y dejándolo reposar en una hamaca:

Estaba cada uno de ellos en un lío luengo, cubierto la cobertura de encima de unas hamacas de paja muy primas e muy bien labradas $[\ldots]$ e mas dentro otro envoltorio e cobertura de mantas mas delgadas e mas primas [...] (citado en Lothrop 1937:46).

Evidencias de la presencia de fuego han sido identificadas en El Caño (Mayo y Mayo 2013:14), Sitio Conte (Lothrop 1937:52) y Cerro Juan Díaz (Cooke 2001:58). En este último sitio grandes 
cantidades de residuos quemados han sido descritos y se han interpretado como hornos mortuorios. Aunque desconocemos cuál fue exactamente el procedimiento de ahumado de los cadáveres, probablemente fuese lento y habría exigido un aprovisionamiento constante de leña durante todo el proceso. Otro tipo de tratamiento post mortem del difunto podría ser el identificado en el guerrero principal de la tumba T2 de El Caño que fue desecado antes de ser amortajado con bandas empapadas de resinas calientes, como se deduce de las deformaciones que presentaban varios de los elementos de su ajuar que se relacionan con la aplicación de calor y la acción de envolver el cuerpo (Mayo y Mayo 2013; Mayo y Carles 2015).

En definitiva, tanto las evidencias etnohistóricas como las arqueológicas permiten afirmar la importancia de la madera en diferentes facetas del ritual funerario (como combustible para el tratamiento de los cadáveres, como elemento de construcción de las estructuras funerarias y como elementos de ajuar). Por ello su estudio es fundamental para la caracterización de los diversos elementos del ritual funerario.

\section{Material y Métodos}

Los conjuntos arqueobotánicos analizados fueron recuperados durante la campaña de excavación del año 2008, que se centró en los depósitos más superficiales. Por este motivo la estrategia de muestreo fue orientada a aquellos contextos mejor preservados y que podrían potencialmente proporcionar material carbonizado, procurando evitar contextos afectados por procesos postdeposicionales relacionados con las inundaciones periódicas del sitio.

Los fragmentos de carbón de mayor tamaño fueron recogidos a mano y de forma individual durante la excavación, con el objetivo de prevenir su fragmentación y evitar la sobrerrepresentación de aquellos taxones que producen carbones de mayores dimensiones (Figura 4), fueron seleccionadas $10 \mathrm{de}$ las muestras puntuales recogidas. Siete muestras de sedimento (160,05 litros) fueron procesadas mediante cribado en seco utilizando mallas de 2 y $1 \mathrm{~mm}$, y todas fueron positivas en la presencia de carbón, excepto la muestra recuperada en la UE046 que fue negativa. De las muestras cribadas cinco fueron seleccionadas para análisis.

En total 15 muestras de los contextos mejor preservados y que podrían aportar información paleoetnobotánica acerca del uso de los recursos forestales en el sitio arqueológico fueron seleccionadas para el análisis antracológico. Se identificaron 215 fragmentos de carbón. Cuando fue posible se analizaron 20 fragmentos por muestra, aunque en algunos casos no fue posible alcanzar esa cifra.

La mayor parte de los fragmentos estudiados $(71,2 \%)$ proceden del Conjunto 5 (Figura 5), que agrupa varias estructuras tipo fosa alguna con los restos de postes carbonizados in situ que parecen delimitar un espacio funerario, uno de los fragmentos (UE050) fue datado en el 631-770 cal. d.C. (Beta244670, 1340 \pm 40 a.p., carbón, calibrado con OxCal v.4.2.2) (Mayo y Mayo 2013). El resto de fragmentos $(28,8 \%)$ estaban dispersos en los depósitos más recientes de esta área (UE006, UE010). En la UE006, interpretada como un nivel de ocupación, se recuperaron fragmentos de cerámica, puntas de proyectil, fragmentos de una figura de tumbaga, un caparazón de tortuga y varias piezas de oro. Y la UE010 era un depósito de sedimento con una gran concentración de cerámicas, una punta de proyectil, una concha, un fragmento de hueso y el fragmento de un artefacto de oro.

Las muestras de carbón fueron observadas en un microscopio de luz reflejada con objetivos de $2 \mathrm{x}, 4 \mathrm{x}$, 20x y 40x y fotografiadas utilizando un microscopio electrónico de barrido (ZEISS EVO LS 15 con EDX). La identificación taxonómica se llevó a cabo mediante fractura manual siguiendo los tres planos anatómicos de la madera: transversal, tangencial y radial. La complejidad de la identificación hizo que los fragmentos fueran agrupados en taxones (p.ej., taxón indeterminado A, B, C, etc.), que en algunos

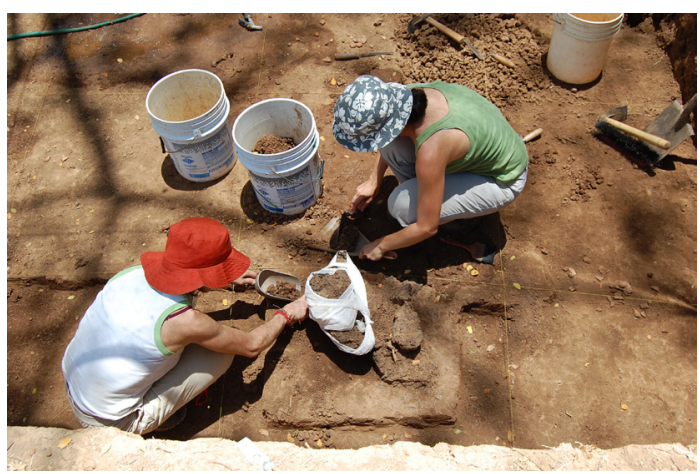

Figura 4. Proceso de extracción de uno de los postes carbonizados y preservados in situ.

Process of extraction of one of the carbonized posts that was preserved in situ. 


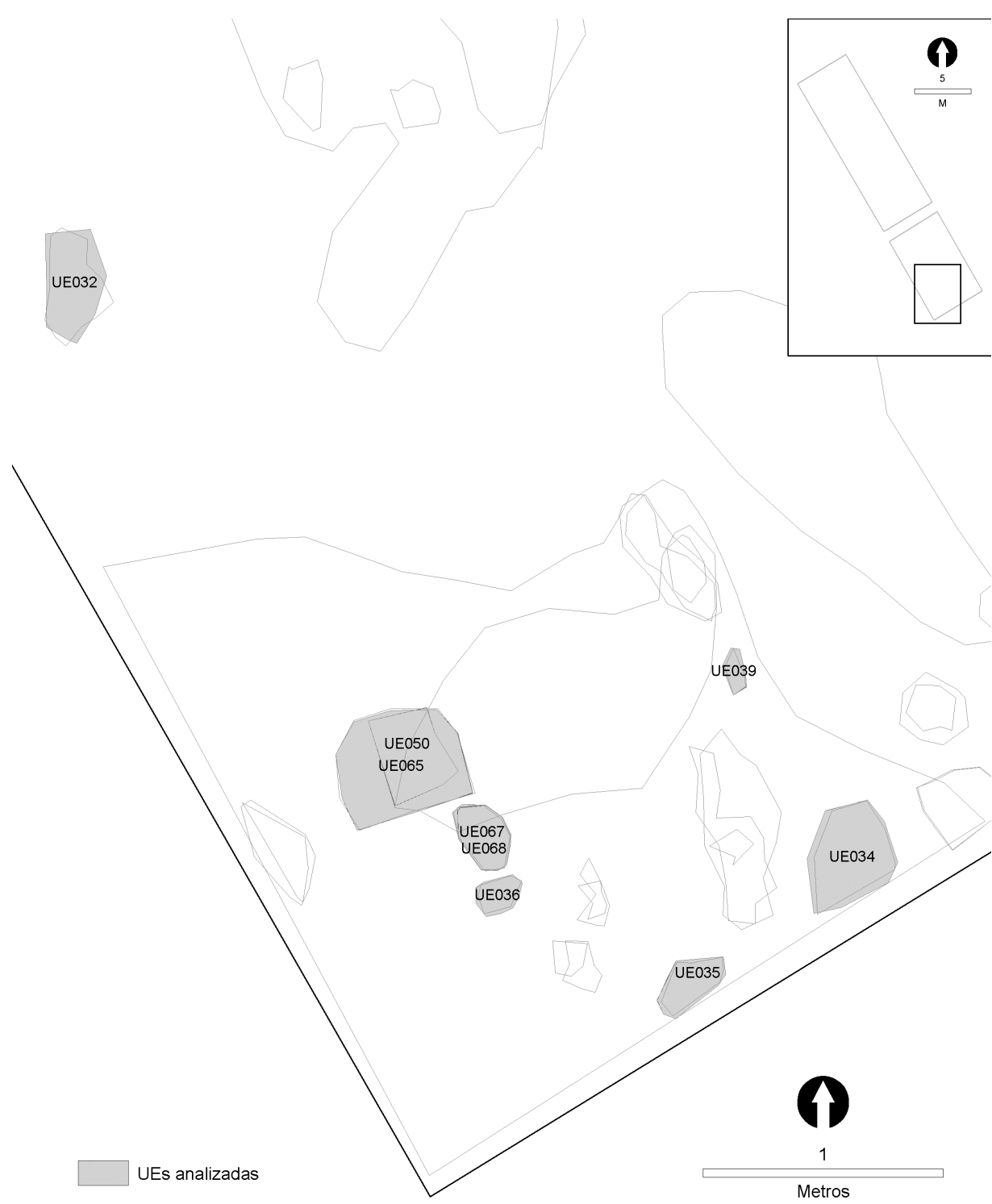

Figura 5. Unidades estratigráficas analizadas en el Conjunto 5. En la imagen aparecen las dos áreas de excavación abiertas durante la campaña del 2008, y los contornos de varias unidades estratigráficas.

Stratigraphic units analyzed in Assemblage 5. View of the two excavation areas opened during the 2008 campaign, and the outline of various stratigraphic units.

casos pudieron ser posteriormente identificados mediante comparación con varias bases de datos (InsideWood.2004-onwards; Richter y Dallwitz 2002), atlas de anatomía de maderas (Carpio 2003, Espinoza y Melandri 2000, Espinoza y León 2001, León 2002, León y Espinoza 2001), publicaciones especializadas (Espinoza y Melandri 1999-2000; Lens et al. 2005; León 2001; León 2014; Kukachka 1981; Oskolski 1995) y con nuestra colección de referencia.
De forma paralela a la identificación se registraron varias características dendrológicas (Marguerie y Hunot 2007; Théry-Parisot et al. 2010) con el objetivo de caracterizar el tipo de consumo de los recursos leñosos: parte de la planta consumida (tallo, rama, raíz) y/o la parte del tallo (duramen o albura), además de otras alteraciones que pueden proporcionar información respecto del proceso de combustión, las condiciones de crecimiento y los procesos tafonómicos. 
Tabla 1. Resultados de la identificación taxonómica de las muestras del Conjunto 5.

Results of charcoal analysis from the samples of Assemblage 5.

\begin{tabular}{|c|c|c|c|c|c|c|c|c|c|c|}
\hline \multicolumn{2}{|c|}{ Conjunto 5} & \multicolumn{9}{|c|}{ Unidad estratigráfica } \\
\hline Taxón & Nombre común & 32 & 34 & 35 & 36 & 39 & 50 & 65 & 67 & 68 \\
\hline Rhizophora sp. & Mangle & & 3 & & 6 & 20 & 20 & 20 & 10 & 5 \\
\hline cf. Manilkara sp. & Níspero & 3 & & & & & & & & \\
\hline Schefflera tipo D1 & Mangavé & & 2 & & & & & & & \\
\hline Pelliciera rhizophorae & Mangle piñuelo & & 2 & & & & & & & \\
\hline Taxón indeterminado F & - & & & 53 & & & & & & \\
\hline Taxón indeterminado $\mathrm{H}$ & - & & & 1 & & & & & & \\
\hline Taxón indeterminado I & - & & 2 & 1 & & & & & & \\
\hline Monocotiledónea & - & & & & & & & & & 5 \\
\hline Núm. de fragmentos & & 3 & 9 & 55 & 6 & 20 & 20 & 20 & 10 & 10 \\
\hline
\end{tabular}

\section{Resultados}

El conjunto de carbones estudiado permitió diferenciar once taxones en total, que en siete casos pudieron ser identificados a nivel de especie, género o familia (Tablas 1 y 3). Si observamos la distribución de estos taxones en relación con los contextos arqueológicos de procedencia (Figura 6), vemos cómo en el Conjunto 5, en el que se concentraban varias estructuras escavadas tipo fosa situadas próximas unas a otras, hay un claro predominio de la madera de mangle (Rhizophora sp.) (Tabla 1, Figura 7). En cinco de las unidades estratigráficas el mangle fue el único taxón identificado, y en otro de los casos (UE068) estaba acompañado de varios fragmentos de monocotiledónea (Figura 8). Estos últimos presentaban en todos los casos las aristas redondeadas.

Los fragmentos de mangle presentaban curvatura débil en el anillo y presencia de tílides, por lo que los carbones se corresponderían con madera del duramen, y la parte de la planta explotada probablemente serían troncos o grandes ramas (Tabla 2). En todos los casos los fragmentos analizados presentaban aristas angulosas, correspondientes con fracturas recientes.

En la UE034 acompañaban al mangle otros taxones como el mangavé (Schefflera tipo D1) (Figura 9), el mangle piñuelo (Pelliciera rhizophorae) (Figura 10) y el Taxón indeterminado I (Figura 11). Finalmente, en las dos unidades estratigráficas que más se diferenciaban de las anteriores el mangle estaba ausente, identificándose en la UE032 la
Tabla 2. Curvatura del anillo de crecimiento en los carbones recuperados del interior de las estructuras del Conjunto 5 .

Tree-ring curvature of charcoal recovered from the infilling of the structures grouped in Assemblage 5.

\begin{tabular}{lcccccc}
\hline Curvatura/UE & 36 & 39 & 50 & 65 & 67 & 68 \\
\hline $\begin{array}{l}\text { Débil } \\
\text { Moderada }\end{array}$ & 6 & 20 & 20 & 15 & 10 & 5 \\
$\begin{array}{l}\text { Fuerte } \\
\text { Sin datos }\end{array}$ & & & & & & \\
\hline
\end{tabular}

Tabla 3. Resultados de la identificación taxonómica de los carbones dispersos en depósitos.

Results of charcoal analysis from scattered charcoal.

\begin{tabular}{lrr}
\hline Taxón/Unidad estratigráfica & 6 & 10 \\
\hline cf. Manilkara sp. & 40 & \\
Fabaceae & & 11 \\
Taxón indeterminado D & 1 \\
Taxón indeterminado E & 9 \\
Indeterminable & & 1 \\
Número de fragmentos & 40 & 22 \\
\hline
\end{tabular}

presencia de níspero (cf. Manilkara sp.) (Figura 12) y en la UE035 los taxones indeterminados $\mathrm{F}$ (Figura 13), H (Figura 14) e I.

En cuanto a los carbones recuperados dispersos en depósitos arqueológicos, de los que es más difícil establecer su origen y por tanto son de más difícil interpretación, se identificó níspero en la UE006 y en la UE010 un taxón de la familia Fabaceae (Figura 15), y los taxones indeterminados D (Figura 16) y E (Figura 17) (Tabla 3). 


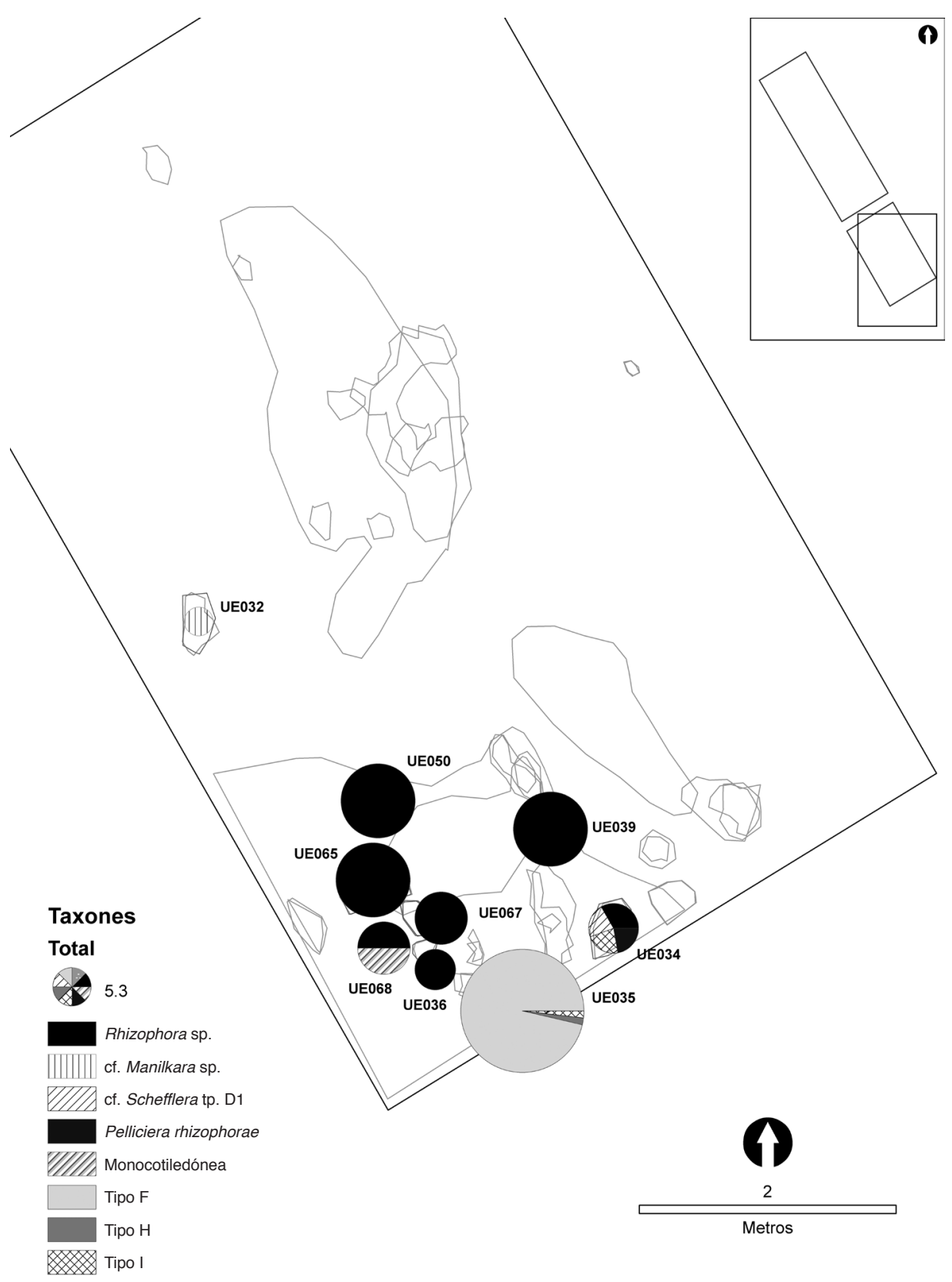

Figura 6. Distribución espacial de los taxones en el Conjunto 5. Spatial distribution of the taxa in the Assemblage 5.

\section{Discusión}

Las maderas carbonizadas recuperadas del interior de las estructuras del Conjunto 5 de El Caño se corresponderían muy probablemente con los restos de los postes originales de una construcción y en ellos fue identificada madera de mangle de forma recurrente. Los datos dendrológicos apuntarían a que se utilizó el duramen de troncos del género Rhizophora. En el único caso en que se recuperaron asociados a la madera de mangle fragmentos de monocotiledónea, estos probablemente se encontraban en posición secundaria y habían sido afectados por procesos de movilización, ya que presentaban las aristas redondeadas. Entre las monocotiledóneas se incluye una gran cantidad de 

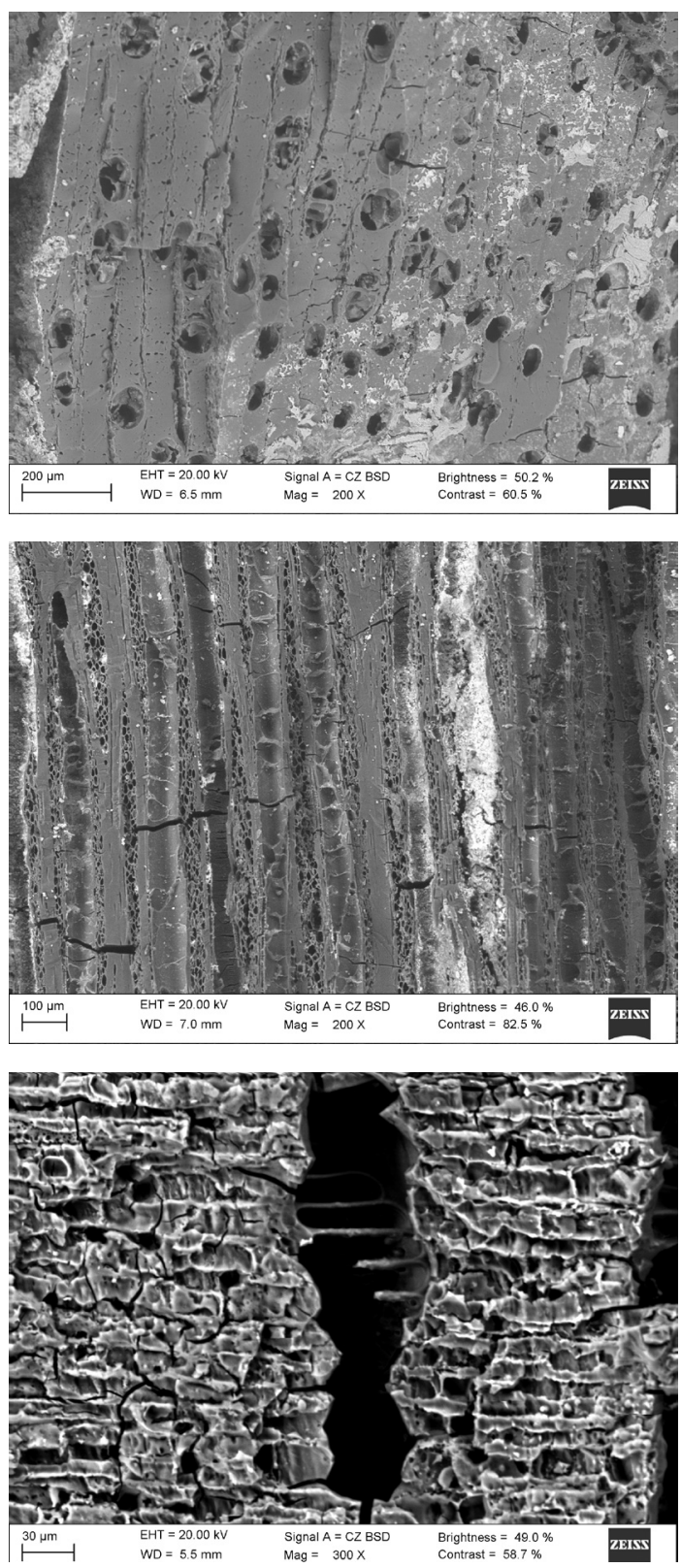

Figura 7. Mangle, Rhizophora sp., Rhizophorae.

Mangrove, Rhizophora sp., Rhizophorae.

plantas, entre ellas palmas como las utilizadas en la cubierta de los bohíos.

Estos datos apuntarían a que en el área que delimita la zona de las tumbas existió una estructura conformada por varios postes, que podría corresponderse con una edificación tipo bohio (Fuson 1964) como las descritas por Fernández de Oviedo, similares a las que se conservan en la actualidad entre los Gunas de Archipiélago de Guna Yala (Figura 18)

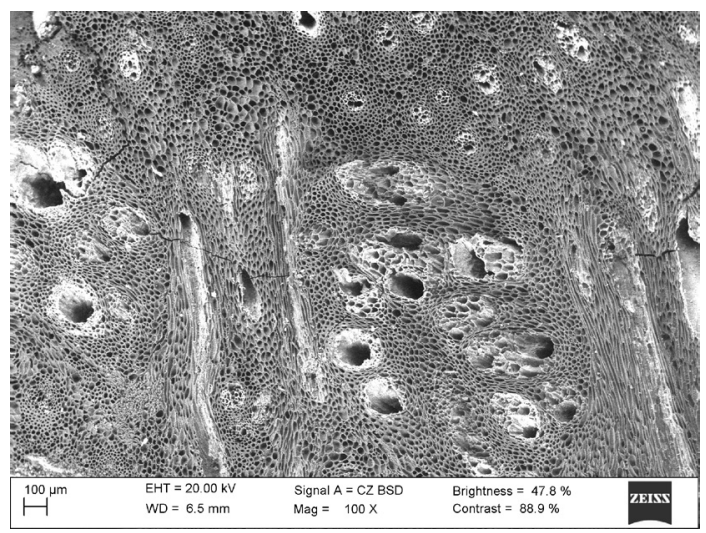

Figura 8. Monocotyledoneae.

Monocotyledoneae.

y de las que existen referencias etnohistóricas en otras áreas americanas (Langebaek 1998). Los postes de madera de estas construcciones estaban cargados de simbolismo para las comunidades de Centroamérica, Norte de América del Sur y Oceanía, existiendo numerosas referencias al respecto (Coiffier y Orliac 2000, 2002; Helms 1979; Howe 1977; Kahn y Coil 2006; Kolb y Murakami 1994; Langebaek 1998). El caso más próximo geográfica y culturalmente es de los Guna. Para este pueblo, los principales postes de sus bohíos se asocian a los jefes y a otros personajes de importancia a nivel ritual o político (Helms 1979). Existiendo una asociación metafórica entre los árboles, los postes de madera, los ríos y el sol con los individuos que detentan la jefatura (Howe 1986:64). Por tanto, la selección de las especies leñosas empleadas tanto para este fin como para la elaboración de otro tipo de objetos era probablemente cuidadosa, ya que sus cualidades derivan de las virtudes de la madera en la que están elaborados (Coiffier y Orliac 2000; Howe 2009:259; Orliac 1990-1).

La selección de madera de mangle para confeccionar los postes del bohío que cubría las tumbas de El Caño probablemente no fue casual, sino intencionada, su madera muy dura y pesada es prácticamente imputrescible (Tengberg 2005). Respecto de la madera de construcción de los bohíos realizada por Gonzalo Fernández de Oviedo, este indica simplemente que se utiliza "reçia e buena madera", señalando en el caso concreto del mangle que:

[...] es un árbol de los mejores que en estas partes hay [...] é para varaçones de 
buhíos e estantes ó postes para las casas [...] es de las mejores maderas que hay acá" (Fernández de Oviedo IX, VI:338).

Desconocemos cuál fue la morfología de los postes del bohío, pero es posible que estuviesen labrados de forma similar a las columnas de los conjuntos escultóricos de El Caño y Sitio Conte (Lothrop 1937:36, 39-40), las vigas de los techos
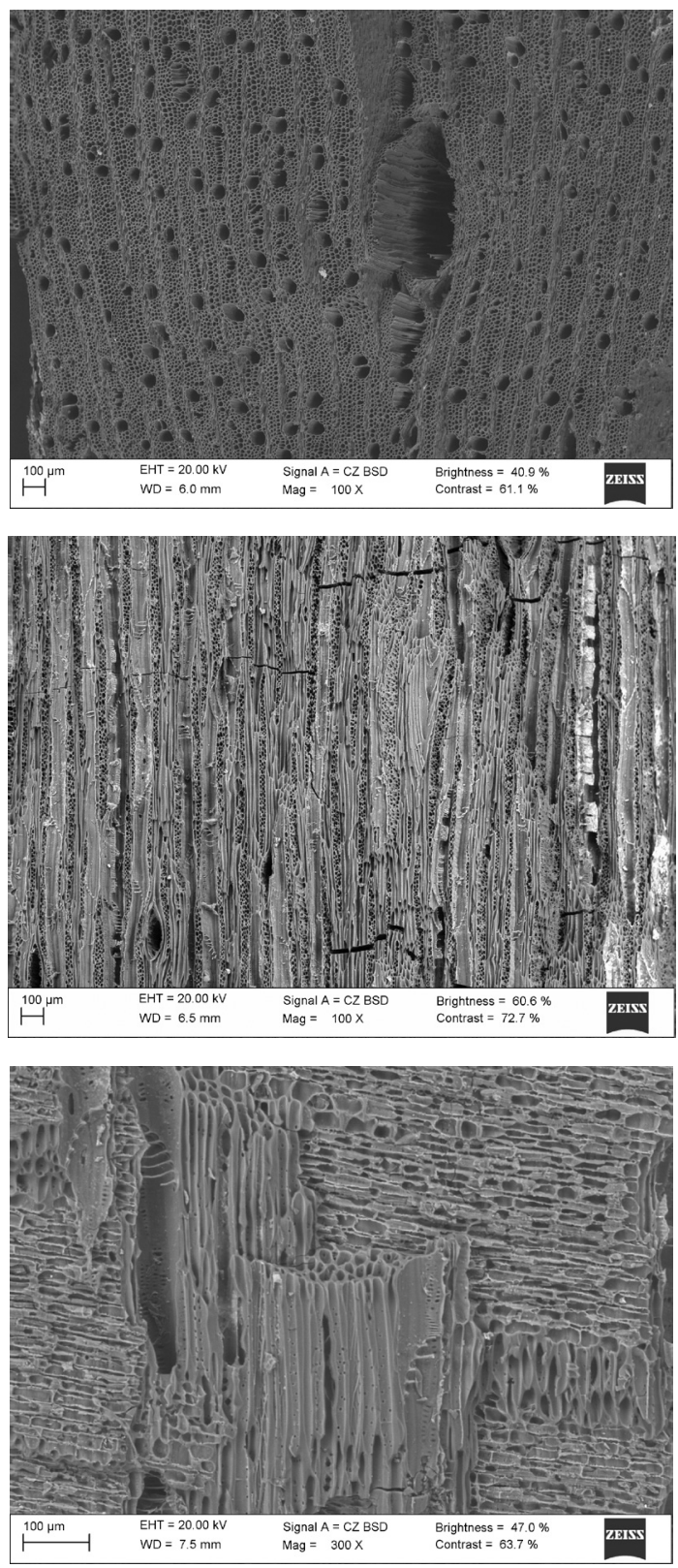

Figura 9. Mangavé, cf. Schefflera tp. D1, Arialaceae. Mangavé, $c f$. Schefflera tp. D1, Arialaceae. también estuvieron probablemente labradas (Helms 1979:9). El estudio realizado previamente sobre las columnas de basalto de El Caño nos permite realizar una aproximación indirecta a la posible morfología de los postes de madera. En las esculturas de basalto fueron identificados dos tipos de trabajos del fuste, en unos casos las columnas son cilíndricas y en otros presentan un formato cuadrangular con esquinas redondeadas.
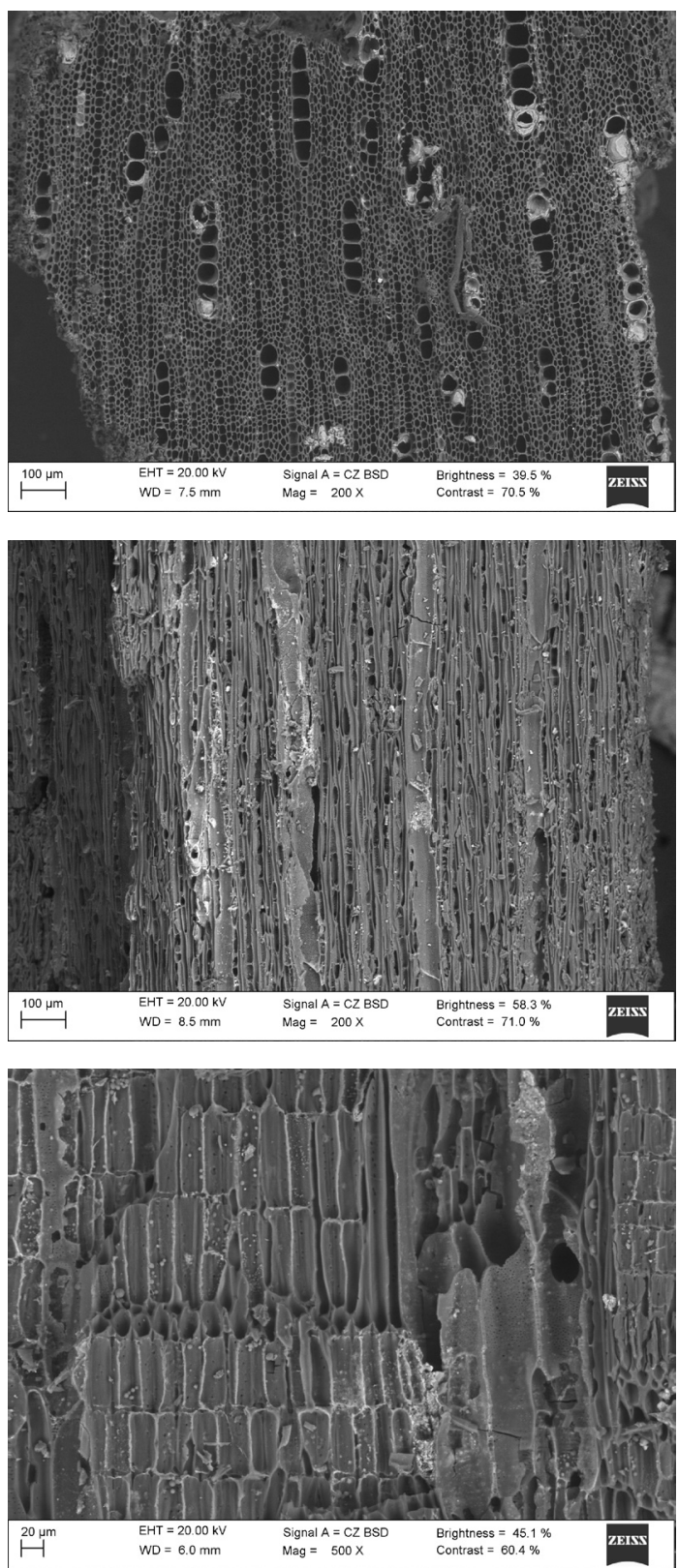

Figura 10. Mangle piñuelo, Pelliciera rhizophorae, Pellicieraceae. Mangle piñuelo, Pelliciera rhizophorae, Pellicieraceae. 

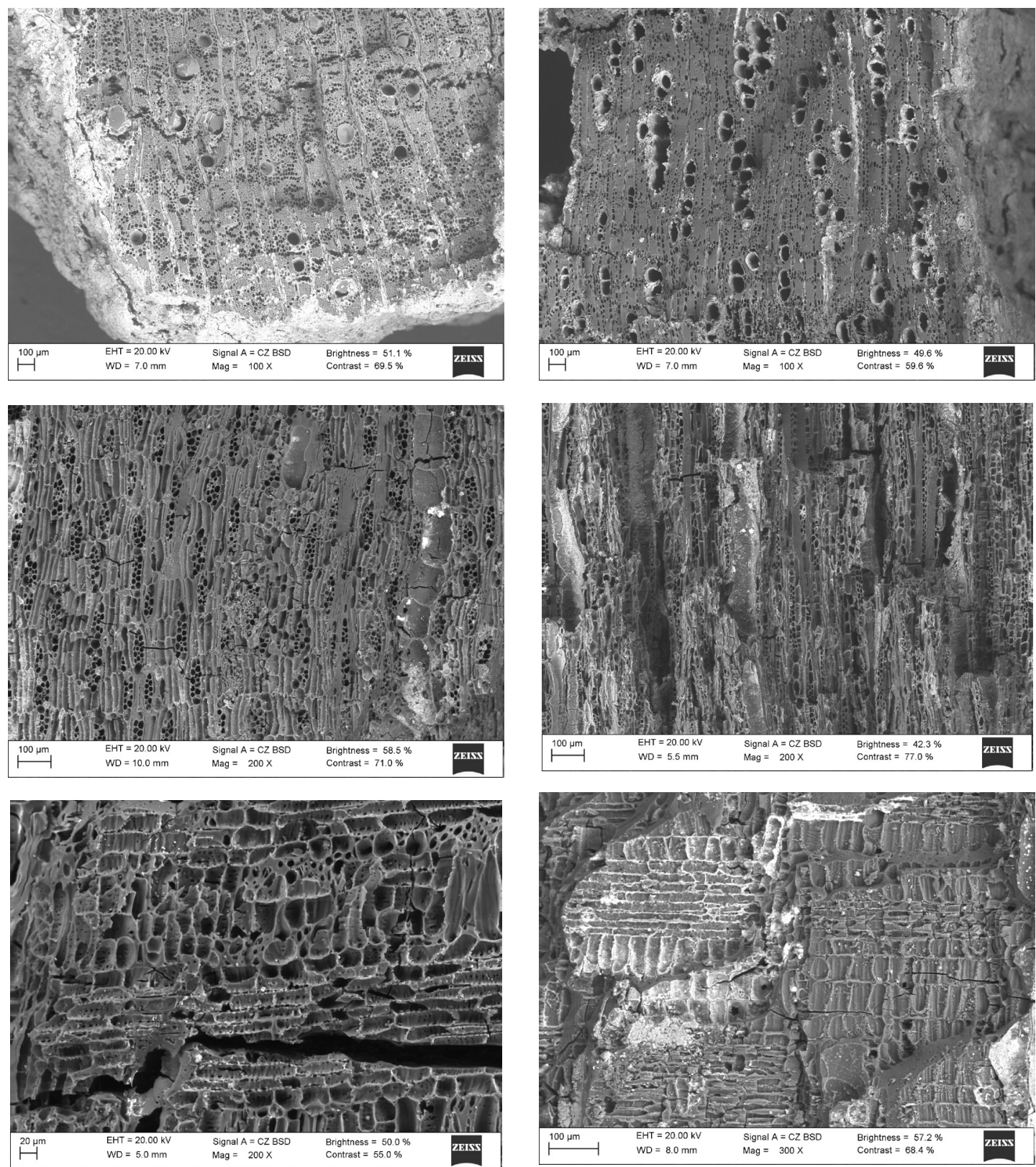

Figura 11. Taxón indeterminado I. Non-identified taxon I.

Figura 12. Níspero, cf. Manilkara sp., Sapotaceae. Noseberry, cf. Manilkara sp., Sapotaceae.

Finalmente, los datos antracológicos de El Caño nos permiten también realizar consideraciones a nivel ambiental y de explotación del territorio por parte de las sociedades de jefatura del Río Grande. Los taxones identificados muestran cómo los Coclé incursionaron en busca de madera en formaciones forestales próximas, la más lejana el manglar. El

bosque de manglar más cercano se encuentra en la boca de Río Grande, y en él pueden encontrarse hoy tanto el mangle (Rhizophora sp.) como el mangle piñuelo (Pelliciera rhizophorae); por su parte, la presencia de níspero (cf. Manilkara sp.), mangavé (cf. Schefflera tipo D1) y Fabaceae indica que además fue explotado también el bosque tropical seco. La 

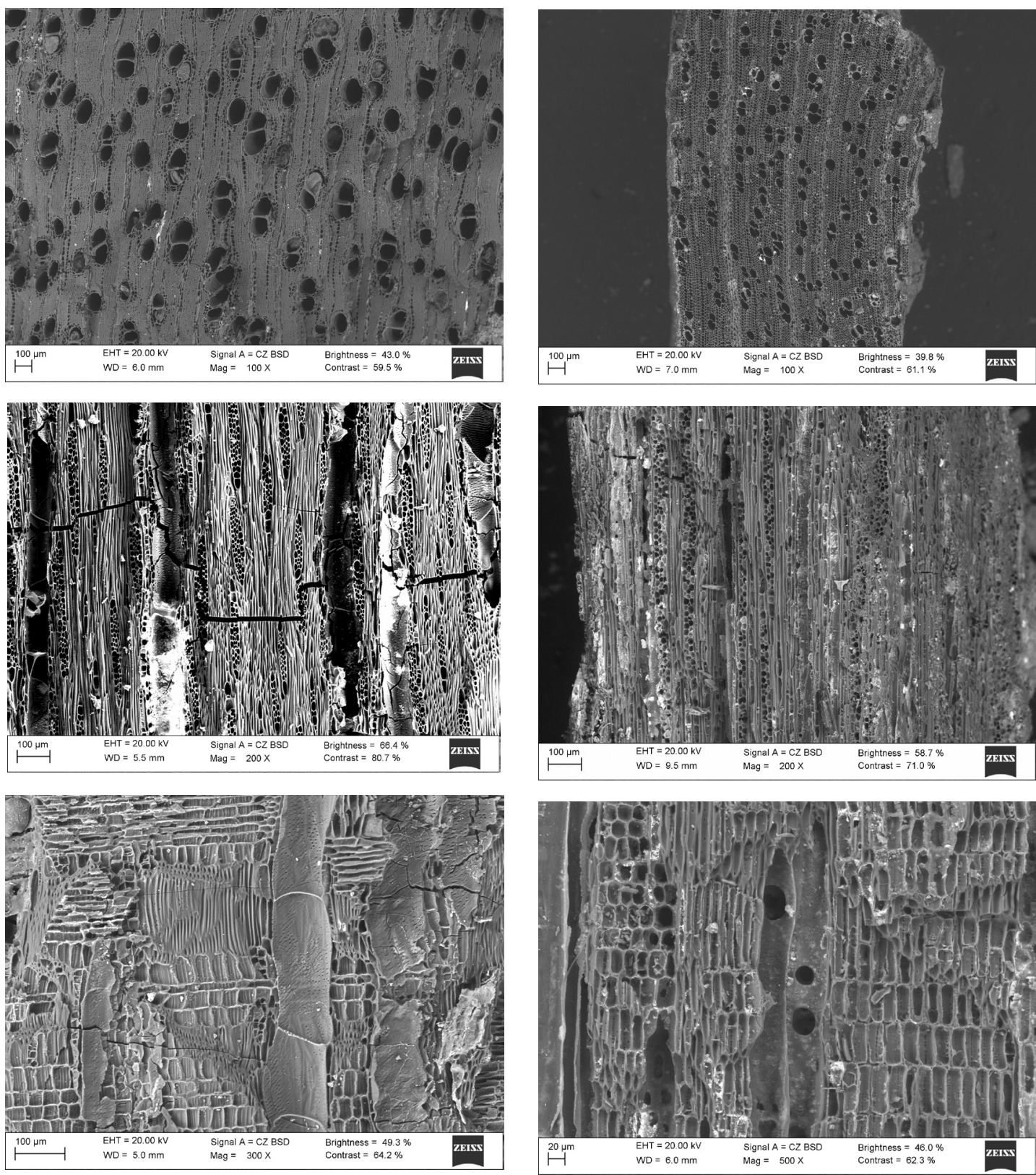

Figura 13. Taxón indeterminado F. Non-identified taxon F.

Figura 14. Taxón indeterminado $\mathrm{H}$. Non-identified taxon $\mathrm{H}$.

obtención de estos recursos leñosos probablemente implicaba una compleja organización del trabajo en diferentes etapas que incluían desde la obtención de la materia prima, al transporte y la preparación de la madera para su uso final; en la que no sería descartable que existieran prohibiciones y tabúes respecto de la tala de árboles (Howe 1986:12).

\section{Conclusiones}

Estos datos antracológicos a pesar de corresponderse con una pequeña parte de las muestras arqueobotánicas recuperadas en El Caño hasta la actualidad muestran las posibilidades que este tipo de análisis ofrece en el estudio de las 

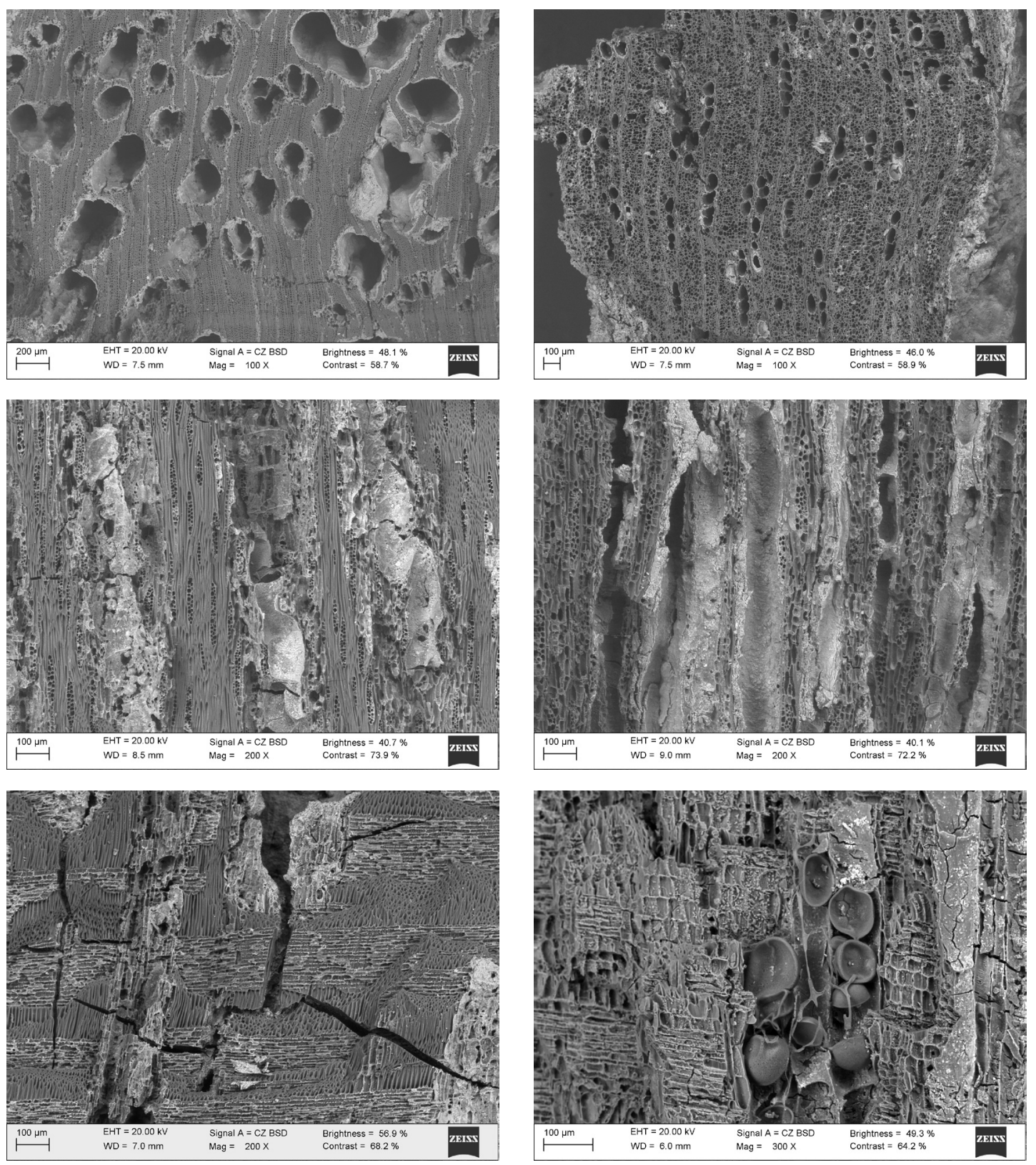

Figura 15. Fabaceae.

Fabaceae.

Figura 16. Taxón indeterminado D.

Non-identified taxon $D$.

jefaturas del Área Intermedia, aportando datos relevantes no solo a nivel ambiental y de gestión de los recursos naturales, sino también sobre las preferencias culturales. Los resultados obtenidos ponen de relieve el papel fundamental que la madera desarrollaba en el ritual funerario y su probable asociación con aspectos simbólicos, de acuerdo con lo documentado en la literatura etnográfica. Pese al potencial de este tipo de estudios, que se refleja en el presente trabajo, también cabe señalar las limitaciones de su aplicación debido a la alta diversidad de especies, la falta de colecciones de referencia y la escasez de estudios publicados acerca de la anatomía de las maderas de la región. 

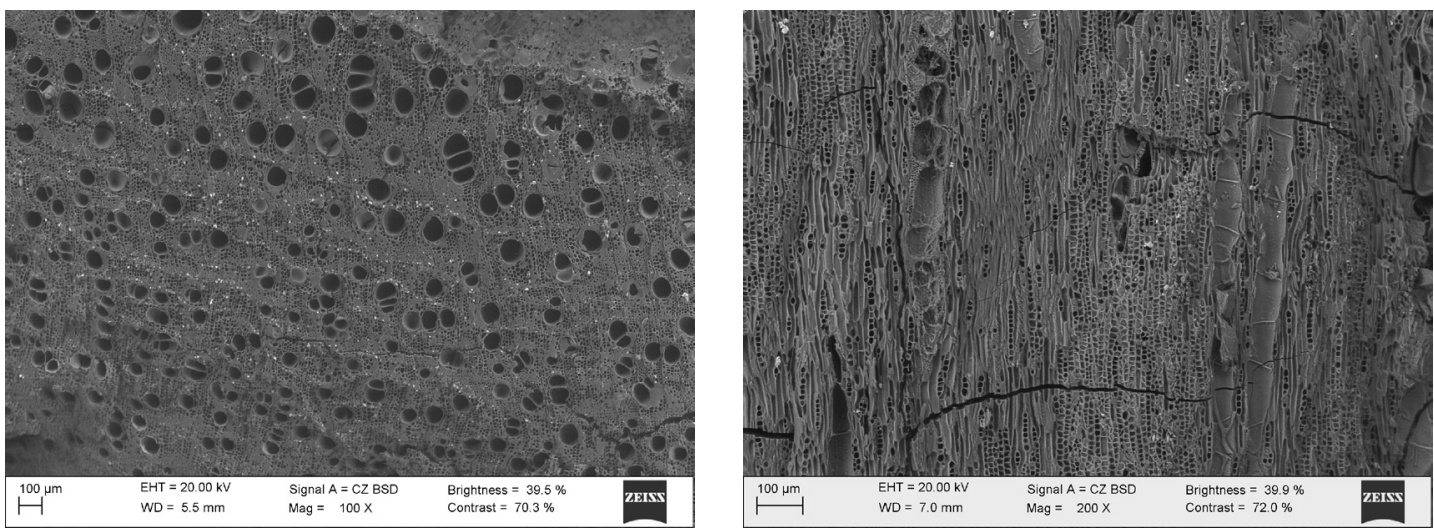

Figura17. Taxón indeterminado E.

Non-identified taxon E.

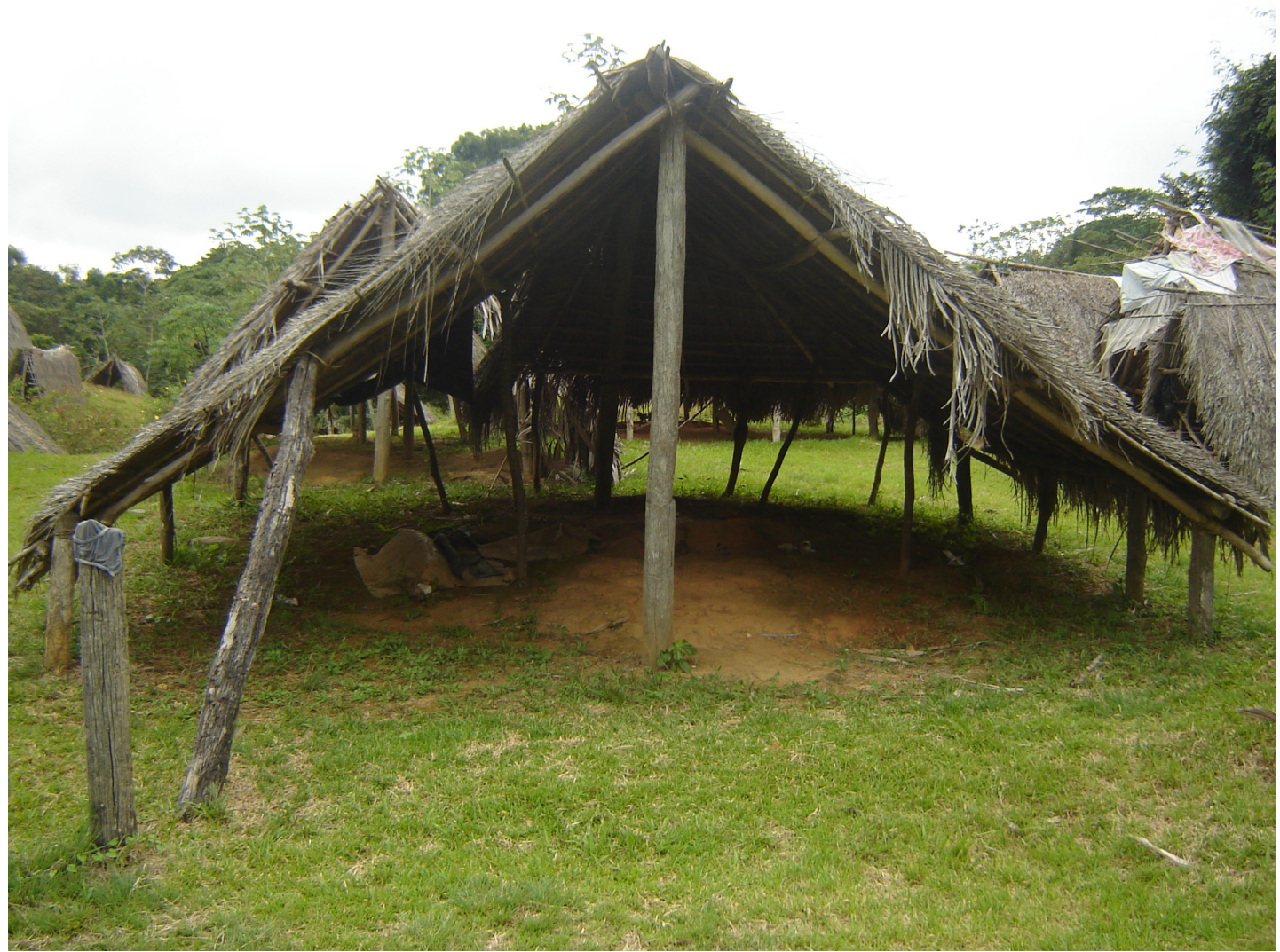

Figura 18. Construcción en madera del cementerio de Cartí en Guna Yala (foto: Ainslie Harrison).

Timber building in the cemetery of Carti in Guna Yala (photo: Ainslie Harrison).

El trabajo demuestra la necesidad de aplicar métodos de recogida de muestras sistemáticos que permitan paliar el vacío de datos antracológicos en esta área.

Agradecimientos: La Fundación El Caño quiere agradecer al Instituto Nacional de Cultura
(INAC), a la Secretaría Nacional de Ciencia y Tecnología (SENACYT) y a la National Geographic Society por su apoyo financiero al proyecto. Al Smithsonian Tropical Research Institute (STRI) por su vital apoyo, tanto en la logística como en el equipamiento. Al Museum Conservation Institute 
(MCI) por su ayuda en la conservación de los artefactos arqueológicos. También agradecemos la asistencia proporcionada por los trabajadores del Parque Arqueológico de El Caño. A la Universidad de Santiago de Compostela por su contribución al proyecto, y la financiación de los desplazamientos de los miembros del equipo adscritos al GEPNGrupo de Estudios para la Prehistoria del NW Ibérico. Finalmente, los autores quieren dejar constancia de su agradecimiento a los evaluadores por sus valiosos comentarios y sugerencias que han permitido mejorar el manuscrito inicial.

\section{Referencias Citadas}

Archila, S. 2008. Modelos teóricos y arqueobotánica en el Noroeste de Suramérica. En Arqueobotánica y Teoría Arqueológica. Discusiones desde Suramérica, compilado por S. Archila, M. Giovanetti y V. Lema, pp. 65-95. Universidad de los Andes, Bogotá.

Archila, S. e I. Cavelier 2006. Hornos, ollas y bosques. Transformación del paisaje por el uso de leña para producción cerámica artesanal en Ráquira, Boyacá. Ponencia presentada al IV Congreso de Arqueología en Colombia, Pereira.

Berman, M.J. y D.M. Pearsall 2000. Plants, people, and culture in the prehistoric central Bahamas: a view from the Three Dog Site, an early Lucayan settlement on San Salvador Island, Bahamas. Latin American Antiquity 65:219-239.

Carpio, I.M. 2003. Maderas de Costa Rica. 150 Especies Forestales. Ed. Universidad de Costa Rica, San José.

Carreras, R. 2009. Las maderas en los objetos aborígenes cubanos. Anales del Museo de América XVII:166-182.

Cavallaro, D. A. 2013. Reconstructing the Past: Paleoethnobotanical Evidence for Ancient Maya Plant Use Practices at the Dos Pilas Site, Guatemala. Dissertation University of Cincinnati.

Coiffier, Ch. y C. Orliac 2000. L'arbre miamba et la maison cérémonielle iatmul (Papouasie Nouvelle-Guinée). Journal d'Agriculture Traditionnelle et de Botanique Appliquée 42:147-164.

Coiffier, Ch. y C. Orliac 2002. Choix symbolique des bois d'œuvre au Vanuatu et en Nouvelle-Guinée. En Le Voyage de la Korrigane dans les Mers du Sud, editado por L. Gauthier, pp. 222-223. Ed. Hazan et Muséum National d'Histoire Naturelle, Paris.

Condit, R., R. Pérez y N. Daguerre 2011. Trees of Panama and Costa Rica. Princeton Field Guides, New Jersey.

Cooke, R.G. 1972. The Archaeology of the Western Coclé Province of Panama. PhD Dissertation. Institute of Archaeology and University of London, London.

Cooke, R.G. 1998. Subsistencia y Economía Casera de los Indígenas Precolombinos de Panamá. Editorial Universitaria, Panamá.

Cooke, R.G. 2001. Cuidando a los ancestros: rasgos mortuorios precolombinos en Cerro Juan Díaz, Los Santos. En Panamá: Puente Biológico, editado por S. Heckadon-Moreno, pp. 54-62. Smithsonian Tropical Research Institute, Panamá.

Cooke, R. G. 2004a. Observations on the Religious Content of the Animal Imagery of the 'Gran Coclé' Semiotic Tradition of Pre-Columbian Panama. En Behaviour Behind Bones. The Zooarchaeology of Ritual, Religion, Status and Identity, editado por S. O’Day, W. Van Neer y A. Ervynck, pp. 114-127. Oxbow Books, Oxford.

Cooke, R. G. 2004b. Rich, Poor, Shaman, Child: Animals Rank and Status in the 'Gran Cocle' Culture Area of Pre-Columbian Panama. En Behaviour Behind the Bones. The Zooarchaeology of Ritual, Religion, Status and Identity, editado por S. O'Day, W. Van Neer y A. Ervynck, pp. 271-284. Oxbow Books, Oxford.

Cooke, R.G. y L.A. Sánchez 2004. Panamá prehispánico. Historia General de Panamá 1 (2):3-89.

Descola, P. 1998. Las cosmologías de los indios de la Amazonia. Zainak 17:219-227.

Espinosa, G. de 1864. Relación hecha por Gaspar de Espinosa, Alcalde Mayor de Castilla del Oro, dada á Pedrárias de Avila, Lugar Teniente General de aquellas provincias, de todo lo que sucedió en la entrada que hizo en ellas, de órden de Pedrárias. En Colección de Documentos Inéditos Relativos al Descubrimiento, Conquista y Colonización de las Posesiones Españolas en América y Oceanía, Sacados en su Mayor Parte del Real Archivo de Indias, compilado por J.F. Pacheco, F. De Cárdenas, L. Torres de Mendoza, tomo II, pp. 467-522. Imprenta Española, Madrid.

Espinoza, N. y W.J. León 2001. Atlas para la Identificación Microscópica de Maderas Latifoliadas. Universidad de los Andes, Mérida.

Espinoza, N. y J.L. Melandri 1999-2000. Estudio anatómico del leño de cinco especies comerciales de la Subfamilia Mimosoideae en Venezuela. Pittieria 29-30 (1):75-88.

Espinoza, N. y J.L. Melandri 2000. Anatomía del Tallo de las Monocotiledóneas. Universidad de los Andes, Mérida.

Fernández de Oviedo, G. 1992. Historia General y Natural de las Indias. 5 vols. Biblioteca de Autores Españoles, Madrid.

Fitzgerald, C. 1992. Informe preliminar sobre excavaciones arqueológicas en El Caño (NA-20), temporada 1988. En El Caño: Comunidad y Cultura, pp. 33-79. Centro Subregional de Restauración OEAINAC/Editorial Mariano Arosemena. Panamá.

Fuson, R.H. 1964. House Tipes of Central Panama. Annals of the Association of American Geographers 54 (2):190-208.

Helms, M.W. 1979. Ancient Panama. Chiefs in Search of Power. University of Texas Press, Austin and London.

Heyden, D. 1993. El árbol en el mito y en el símbolo, Estudios de Cultura Náhuatl 23:201-219.

Hood, A.N. 2012. Testing the Veracity of Paleoethnobotanical Macroremain Data: A Case Study from the Cerén Site, El Salvador. Dissertation University of Cincinnati. 
Howe, J. 1977. Carrying the village: Cuna political metaphors. The social use of metaphor. Essays on The Anthropology of Rhetoric, editado por J. D. Sapir y Ch. Crocker, pp. 132-163. University of Pennsylvania Press, Philadelphia.

Howe, J. 1986.The Kuna Gathering: Contemporary Village Politics in Panama. Latin American monographs. University of Texas Press, Austin.

Howe, J. 2009. Chiefs, Scribes, and Ethnographers. Kuna Culture form Inside and Out. University of Texas Press, Austin.

Insidewood. 2004-onwards http://insidewood.lib.ncsu.edu/ search (agosto de 2015).

Kahn, J.G. y J. Coil 2006. What house posts tell us about status difference in prehistoric Tahitian society: An interpretation of charcoal analysis, sacred woods and inter-site variability. The Journal of the Polynesian Society: 319-352.

Kolb, M.J. y G.M. Murakami 1994. Cultural dynamics and the ritual role of Woods in Pre-Contact Hawi'i. Asian Perspectives 33 (1):57-78.

Kukachka, B. F. 1981.Wood anatomy of the neotropical Sapotaceae. XX. Manilkara. USDA Forest Products Laboratory. Research Paper FPL 371.

Langebaek, C. H. 1998. Entierros prehispánicos en viviendas: un ensayo de interpretación. Boletín de Arqueología 2:3-10.

Lens, F., S. Dressler, S. Jansen, L. Van Evelghem y E. Smets 2005. Relationships within Balsamionid Ericales: A wood anatomical approach. American Journal of Botany 92 (6):941-953.

Lentz, D.L., M.P. Beaudry-Corbett, M.L.R. de Aguilar y L. Kaplan 1996. Foodstuffs, forests, fields, and shelter: a paleoethnobotanical analysis of vessel contents from the Ceren site, El Salvador. Latin American Antiquity 61:247-262.

Lentz, D.L. y B. Hockaday 2009. Tikal timbers and temples: Ancient Maya agroforestry and the end of time. Journal of Archaeological Science 36:1342-1353.

León, W.J. 2001. Anatomía del leño, aspectos ecológicos y filogenia en mangles de Venezuela. Revista Forestal Venezolana 45 (2):191-203.

León, W.J. 2002. Anatomía e Identificación Macroscópica de Maderas. Universidad de los Andes, Mérida.

León, W.J. 2014. Anatomía de Maderas de 108 Especies de Venezuela. Revista Pittieria. Número especial:1-267.

León, W.J. y N. Espinoza 2001. Anatomía de la Madera. Universidad de los Andes, Mérida.

Linares, O.F. 1976. Animals that were bad to eat were good to compete with: An analysis of the Conte Style from Ancient Panama. En Ritual and Symbol in Native South America, editado por P.D. Young y J.D. Howe, Anthropological Papers $N^{\circ} 9$. pp. 120. University of Oregon Press, Eugene.

Linares, O.F .1977. Ecology and the arts in ancient Panama: on the development of social rank and symbolism in the central provinces. Studies in Pre-Columbian art and Archaeology 17:1-86.

Lothrop, S. K. 1934. Archaeological Investigation in the Province of Coclé, Panamá. American Journal of Archaeology 38:207-211.

Lothrop, S. K . 1937. Coclé: An Archaeological Study of Central Panama. Historical Background Excavations at the Sitio Conte:
Artifacts and Ornaments. Part 1. Memoirs of the Peabody Museum of Archaeology and Ethnology 7, Cambridge.

Marguerie, D. y J.-Y. Hunot 2007. Charcoal analysis and dendrology: data from archaeological sites in north-western France. Journal of Archaeological Science 34:1417-1433.

Mayo, J. y J.A. Carles (eds.) 2015. Guerreros de Oro: Los Señores de Coclé. Ed. Caribe, Panamá.

Mayo, J. y C. Mayo 2013. El descubrimiento de un cementerio de élite en El Caño: indicios de un patrón funerario en el valle del Río Grande, Coclé, Panamá. Arqueología Iberoamericana 20:3-27.

Mayo, J., A. Mojica, A. Ruiz, E. Moreno, C. Mayo y G.I. De Gracia 2007. Estructuras arquitectónicas incipientes y áreas de explotación minera prehispánica de las cuencas de los ríos Grande y Coclé del Sur, Panamá. En Gran Coclé: Paisaje Cultural del Istmo de Panamá, editado por J. Mayo. Revista Española de Antropología Americana 37/1:93-110.

McKillop, H. 2005. Finds in in Belize document Late Classic Maya salt making and canoe transport. PNAS 102:5630-5634.

Menzies, A.C.J. y M.J. Haller 2012. A macroregional perspective on chiefly cycling in the Central Region of Panama during the Late Ceramic II Period (A.D. 700-1522). Latin American Antiquity 23:449-466.

Morehart, Ch.T., D.L. Lentz y K.M. Prufer 2005. Wood of the gods: the ritual use of pine (Pinus spp.) by the ancient lowland Maya. Latin American Antiquity 16:255-274.

Morehart, Ch.T. y S. Morell-Hart 2013. Beyond the ecofact: Toward a social paleoethnobotany in Mesoamerica. Journal of Archaeological Method and Theory. DOI: 10.1007/ s10816-013-9183-6.

Murphy, P.G. y A.E. Lugo 1986. Ecology of Tropical Dry Forest. Annual Review of Ecology and Systematics 17:67-88.

Newsom, L.A. y E.S. Wing 2004. On Land and Sea: Native American Uses of Biological Resources in the West Indies. University of Alabama Press, Tuscaloosa.

Orliac, C. 1990-1. Des arbres et des dieux: matériaux de sculpture en Polynésie. Journal de la Société des Océanistes 90 (1):35-42.

Oskolski, A. A. 1995. Wood anatomy of Scheffleria and related taxa (Arialaceae). IAWA Journal 16:159-190.

Ostapkowicz, J., Ch. Bronk Ramsey, F. Brock, T. Higham, A.C. Wiedenhoeft, E. Ribechini, J.J. Lucejko y S. Wilson 2012. Chronologies in wood and resin: AMS 14C dating of preHispanic Caribbean wood sculpture. Journal of Archaeological Science 39:2238-2251.

Ostapkowicz, J., Ch. Bronk Ramsey, A.C. Wiedenhoeft, F. Brock, T. Higham y S.M. Wilson 2011. This relic of antiquity' Fifth to fifteenth century wood carvings from the southern Lesser Antilles. En Communities in contact. Essays in Archaeology, Ethnohistory \& Ethnography of the Amerindian Circum-Caribbean, editado por C.L. Hofman y A. van Duijvenbode, pp. 137-170. Sidestone Press, Leiden.

Ostapkowicz, J., A. Wiedenhoeft, Ch.B. Ramsey, E. Ribechini, S.M. Wilson, F. Brock y T. Higham 2011. 'Treasures. . of black wood, brilliantly polished': five examples of Taíno sculpture from the tenth-sixteenth century Caribbean. Antiquity 85:942-959. 
Pagán, J.R. 2002. Agricultura precolombina de las Antillas: retrospección y análisis. Anales de Antrolopogía 36:43-91.

Pennington, R.T., M. Lavin y A. Oliveira-Filho 2009. Woody plant diversity, evolution, and ecology in the Tropics: Perspectives from seasonally Dry Tropical Forests. Annual Review of Ecology, Evolution, and Systematics 40:437-457.

Prufer, K.M. y P.S. Dunham 2009. A shaman's burial from an Early Classic cave in the Maya Mountains of Belize, Central America. World Archaeology 41:295-320.

Reclus, A. 1881. Panama et Darien. Voyages d'Exploration (1876-1878). Ed. Hachette, Paris.

Richter, G. y M. Dallwitz 2002. Commercial timbers: descriptions, illustrations, identification, and information retrieval. http:// biodiversity.uno.edu/delta/wood/es (consultado agosto de 2015).

Robinson, M.E. y H. McKillop 2013. Ancient Maya wood selection and forest exploitation: a view from the Paynes Creek salt works, Belize. Journal of Archaeological Science 40:3584-3595.

Robinson, M.E. y H. McKillop 2014. Fuelling the Ancient Maya Salt Industry. Economic Botany 68:96-108.
Saunders, N.J. y D. Gray 1996. Zemís, trees, and symbolic landscapes: three Taino carvings from Jamaica. Antiquity 70:801-812.

Tengberg, M. 2005. Les forêts de la mer. Exploitation et évolution des mangroves en Arabie orientale du Néolithique à l'époque islamique. Paléorient 31:39-45.

Théry-Parisot, I., L. Chabal y J. Chrzavzez 2010. Anthracology and taphonomy, from wood gathering to charcoal analysis. A review of the taphonomic processes modifying charcoal assemblages, in archaeological contexts. Palaeogeography, Paleoclimatology, Palaeoecology 291:142-153.

Verrill, H. 1927. A mystery of the vanished past in Panama: Newly discovered relics of a vanished civilization destroyed by earthquake or volcanic eruption. Illustrated London News 173 (\#4669). Londres.

Viveiros de Castro, E.1996. Os pronomes cosmológicos e o perspectivismo ameríndio. Maná 2:115-144.

Wyatt, A.R. 2008. Gardens On Hills: Ancient Maya Terracing and Agricultural Production at Chan, Belize. PhD Dissertation, University of Illinois, Chicago. 